\title{
Syndrome de Guillain-Barré
}

\author{
Ario Mirian MD MSc, Michael W. Nicolle MD DPhil, Adrian Budhram MD
}

Citation : CMAJ 2021 March 15;193:E378. doi : 10.1503/cmaj.202710-f

Voir la version anglaise de l'article ici : www.cmaj.ca/lookup/doi/10.1503/cmaj.202710

1 Le syndrome de Guillain-Barré (SGB) est la cause la plus fréquente de faiblesse neuromusculaire subaiguë en Amérique du Nord

L'incidence médiane du SGB se situe autour de 1 pour 100000 personnesannées. Ce taux est supérieur chez les personnes âgées (augmentation de $20 \%$ du taux moyen de SGB par tranche d'âge de 10 ans) et chez les hommes ${ }^{1}$. Le SGB est souvent déclenché par des infections (notamment celles causées par Campylobacter jejuni et le virus de la grippe). Lors d'une vaste étude rétrospective publiée en 2013, aucune augmentation du risque de contracter le SGB n'a été détectée après la vaccination ${ }^{2}$.

2

\section{Le SGB se manifeste d'ordinaire par une faiblesse symétrique} ascendante et par une hyporéflexie

Habituellement, la faiblesse apparaît d'abord dans les membres inférieurs ${ }^{3}$. Dans le contexte des soins primaires, les patients présentent le plus souvent des acroparesthésies, suivies d'une faiblesse subaiguë (moins de 4 semaines), symétrique et flasque, accompagnée d'une hyporéflexie (annexe 1, accessible en anglais au www.cmaj.ca/lookup/ doi/10.1503/cmaj.202710/tab-related-content). Il convient de rediriger les patients vers le service des urgences et d'envisager une hospitalisation pour surveiller l'apparition d'une faiblesse progressive, d'une insuffisance respiratoire ou d'une dysautonomie.

\section{3}

\section{Une ponction du liquide céphalorachidien (LCR) permettra d'exclure les pathologies à symptômes similaires avant} d'amorcer un traitement immunomodulateur

La pléiocytose du LCR constitue un signal d'alarme pour infirmer un diagnostic de SGB; elle peut indiquer une infection aux symptômes similaires, comme la maladie de Lyme ou une infection par le VIH, un entérovirus ou le virus du Nil occidental ${ }^{3}$. Un taux élevé de protéines dans le LCR avec une numération normale des globules blancs (dissociation albumino-cytologique) s'observe dans 50\%-70\% des cas de SGB au cours de la première semaine de symptômes ${ }^{3}$.

L'administration d'immunoglobulines intraveineuses (IgIV)

et la plasmaphérèse constituent des traitements efficaces pour accélérer le rétablissement

Le sous-comité sur les normes de qualité de l'American Academy of Neurology, dans sa ligne directrice fondée sur des essais randomisés contrôlés, recommande l'administration d'IgIV ou la plasmaphérèse aux patients non ambulatoires dans les 2-4 semaines suivant l'apparition des symptômes, respectivement (niveau $\mathrm{A})^{4}$. Les 2 traitements sont aussi efficaces l'un que l'autre pour réduire l'incapacité après 4 semaines ${ }^{4}$. L'administration de corticostéroïdes n'est pas recommandée ${ }^{4}$.

5 Près du tiers des patients auront besoin de ventilation artificielle, mais le pronostic à long terme est généralement favorable.

Près de $30 \%$ des patients atteints du SGB auront besoin de ventilation artificielle. Cette dernière est associée à un taux de mortalité de 5\%-12\%, à une récupération plus lente et à une invalidité résiduelle ${ }^{5}$. La récupération commence après le nadir clinique, qui se produit après 2-4 semaines; environ $83 \%$ des patients peuvent marcher sans aide après 6 mois $^{5}$. Les symptômes persistants peuvent inclure une faiblesse dans les jambes, des douleurs et de la fatigue.

Intérêts concurrents : Aucun déclaré.

Cet article a été révisé par des pairs.

Affiliations : Département de neurologie clinique, London Health Sciences Centre, London, Ont.

Propriété intellectuelle du contenu : Il s'agit d'un article en libre accès distribué conformément aux modalités de la licence Creative Commons Attribution (CC BY-NC-ND 4.0), qui permet l'utilisation, la diffusion et la reproduction dans tout médium à la condition que la publication originale soit adéquatement citée, que l'utilisation se fasse à des fins non commerciales (c.-à-d., recherche ou éducation) et qu'aucune modification ni adaptation n'y soit apportée. Voir : https://creativecommons.org/ licenses/by-nc-nd/4.0/deed.fr.

Correspondance : Ario Mirian, ario.mirian@lhsc.on.ca 


\section{Références}

1. Sejvar JJ, Baughman AL, Wise M, et al. Population incidence of Guillain-Barré syndrome: a systematic review and meta-analysis. Neuroepidemiology 2011;36:123-33.

2. Baxter R, Bakshi N, Fireman B, et al. Lack of association of Guillain-Barré syndrome with vaccinations. Clin Infect Dis 2013;57:197-204.

3. Leonhard SE, Mandarakas MR, Gondim FAA, et al. Diagnosis and management of Guillain-Barré syndrome in ten steps. Nat Rev Neurol 2019;15:671-83.

4. Hughes RA, Wijdicks EF, Barohn R, et al.; Quality Standards Subcommittee of the American Academy of Neurology. Practice parameter: immunotherapy for Guillain-Barré syndrome: report of the Quality Standards Subcommittee of the American Academy of Neurology. Neurology 2003;23:736-40.

5. van den Berg B, Storm EF, Garssen MJP, et al. Clinical outcome of GuillainBarré syndrome after prolonged mechanical ventilation. J Neurol Neurosurg Psychiatry 2018;89:949-54. 